UDC 517.9, 519.6

S. Kamouche, H. Guebbai, M. Ghiat, S. Segni

\title{
GENERALIZED QUADRATIC SPECTRUM APPROXIMATION IN BOUNDED AND UNBOUNDED CASES
}

\begin{abstract}
The goal of this paper is to generalize concepts in spectral theory in order to define the quadratic spectrum associated to three bounded linear operators. This concept was initially defined for three matrices. Moreover, we construct a new method of spectral approximation to avoid the problem of spectral pollution. This problem is resolved with the obtention of property U under the norm convergence or the collectively compact convergence. Also, we make numerical tests on the quadratic pencil associated to Schrödinger's operator in order to validate our theoretical results and to show the efficiency of our method.
\end{abstract}

Key words: generalized quadratic spectrum, spectral approximation, property $U$, quadratic pencil

2020 Mathematical Subject Classification: 34L16, 47A10, $47 A 75,45 C 05,65 N 15,93 B 60$

1. Introduction. Spectral pollution is a common phenomenon in the field of applied mathematics. It is a major problem that appears in the resolution of eigenvalue problems, which is occur in many models of quantum mechanics, solid state physics and elasticity theory (see Boulton et al [5]).

The spectral pollution makes finite elements, finite differences, and direct projection methods meaningless when we apply them to eigenvalue problems of an unbounded operator.

This phenomenon is represented by the appearance of eigenvalues that do not converge to the exact values or the absence of a relationship between the approximate values and the true values. Also, we can illustrate this problem in the non-correlation of the eigenvalues of the approximated

(C) Petrozavodsk State University, 2021 
operator with the spectral properties of the initial operator, like the negative eigenvalues which appear for an unbounded positive definite operator (see Llobet et al in [15], [5], Davies et al in [7] and Rappaz et al [16]).

In recent papers of Guebbai, Khellaf et al [8], [11], [12], [13] and in the book of Ahues et al [1], the authors have tried to limit the problem of spectral pollution in different types of spectral problems. The standard spectral problem is defined as follows: Find scalars $\lambda \in \mathbb{C}$ and non zero vectors $x \in \mathcal{X}$ satisfying

$$
A x=\lambda x,
$$

where, $\mathcal{X}$ is a Banach space and $A: \mathcal{X} \rightarrow \mathcal{X}$ is a bounded operator.

This problem has been treated in the context of classical spectral theory which is presented in the details by Ahues et al in [1]. In [1], the authors build a finite-rank approximation of $A$, which transforms the initial problem $A x=\lambda x$ to an eigenvalue matrix problem. Then, they show that the various types of convergence achieve the property $U$ which allows them to avoid spectral pollution. Among the types of convergence proposed by Ahues et al [1], we are interested in the norm convergence and the collectively compact convergence.

In another context, the authors in [8], [11], [12], [13] dealt with the problem of spectral pollution that appears for unbounded operators. To obtain this, they show that the spectrum of one unbounded operator is equal to the generalized spectrum of two bounded operators of the following form:

$$
\operatorname{sp}(A)=\operatorname{sp}(T, S),
$$

where $A$ is an unbounded operator and $T, S$ are two bounded operators.

In the articles [8], [11], [13], they re-study the results of the property $\mathrm{U}$ obtained in the results obtained by Ahues et al in [1], in order to study the generalized spectral problem $T x=\lambda S x$ in the case of the norm convergence studied in [8] and the collectively compact convergence presented in [11], [13].

Recently, the quadratic spectral problems have attracted a lot of attention in various fields of mathematical modeling, such as the dynamic analysis of mechanical systems in acoustics, linear flow stability in fluid mechanics, and the generalization to the infinite dimension of the transistor problem (see Huang et al [10], Tisseur et al [19]).

In this work, we study the quadratic spectral problem of three bounded operators that can be formed as follows: find scalars $\lambda \in \mathbb{C}$ and nonzero 
vectors $x \in \mathcal{X}$ satisfying

$$
\lambda^{2} A x+\lambda B x+C x=0,
$$

where $A, B$ and $C: \mathcal{X} \rightarrow \mathcal{X}$ are bounded operators. This type of problems is known as the quadratic eigenvalues problem. The case of matrices has been well studied by Chen et al [6], Huang et al [10], Tisseur et al [19].

Our aim in this paper is to push the generalization effected by [8], [11], [13] to give an analytical sense for the generalization of the quadratic spectrum of three bounded linear operators, and we show some properties and characteristics of the generalized quadratic spectrum. Also, we build a new spectral approximation method in order to approximate the generalized quadratic spectrum and prove its convergence.

Finally, the numerical tests are applied on the quadratic pencil of Schrödinger's operator. This can be seen as an illustration of unbounded quadratic spectral problem. It has been studied by Bairamov et al [4], [14]. From this quadratic spectral problem of an unbounded operator, we construct an equivalent problem with three bounded operators, which makes our numerical method applicable.

Numerical tests show that our vision allows us to solve the spectral pollution problem for the quadratic eigenvalue problem of an unbounded operator.

2. Generalized quadratic spectrum. Let $\left(\mathcal{X},\|\cdot\|_{\mathcal{X}}\right)$ be a Banach space, $\operatorname{BL}(\mathcal{X})$ be the Banach space of all linear bounded operators defined on $\mathcal{X}$ to itself. Its norm is described as follows:

$$
\forall A \in \operatorname{BL}(\mathcal{X}): \quad\|A\|=\sup _{\|x\|_{\mathcal{X}}=1}\|A x\|_{\mathcal{X}}
$$

For $A, B$, and $C$ in $\operatorname{BL}(\mathcal{X})$, we define the generalized quadratic spectral problem as follows: find $\lambda \in \mathbb{C}$ and $x \in \mathcal{X}-\{0\}$, such that

$$
Q(\lambda) x:=\lambda^{2} A x+\lambda B x+C x=0 .
$$

In the case when $A, B$, and $C$ are matrices, this type of problems is known as the quadratic eigenvalue problem. It was treated by Tisseur et al, Huang al, Chen et al in [6], [10], [19].

Our aim is to generalize the different results obtained in [8], [11], [13] and the studies of the matrix problem in [6], [10], [19]. 
For that, we define:

- The generalized quadratic resolvent set by

$$
\operatorname{re}(A, B, C)=\{\lambda \in \mathbb{C}: Q(\lambda) \text { is invertible and bounded }\}
$$

- The generalized quadratic point spectrum set by

$$
\operatorname{sp}_{p}(A, B, C)=\{\lambda \in \mathbb{C}, \exists x \in \mathcal{X} \backslash\{0\}: Q(\lambda) x=0\}
$$

- The generalized quadratic essential spectrum set by

$$
\operatorname{sp}_{\text {ess }}(A, B, C)=\{\lambda \in \mathbb{C}: Q(\lambda) \text { is injective, not surjective }\}
$$

- The generalized quadratic spectrum set by

$$
\operatorname{sp}(A, B, C)=\operatorname{sp}_{p}(A, B, C) \cup \operatorname{sp}_{e s s}(A, B, C)=\mathbb{C} \backslash \operatorname{re}(A, B, C) .
$$

Also, we define the generalized quadratic resolvent operator associated to $A, B$, and $C$ by the following function:

$$
\begin{aligned}
\mathrm{R}_{\mathrm{Q}}(\cdot): \operatorname{re}(A, B, C) \subset \mathbb{C} & \rightarrow \mathrm{BL}(\mathcal{X}) \\
z & \mapsto \mathrm{R}_{\mathrm{Q}}(z)=Q^{-1}(z)=\left(z^{2} A+z B+C\right)^{-1}
\end{aligned}
$$

And, we define the radius $\kappa(\lambda)$ as follows: For all $\lambda \in \operatorname{re}(A, B, C)$

$$
\begin{aligned}
\kappa(\lambda) & =\frac{\sqrt{\left(\left\|\mathrm{R}_{\mathrm{Q}}(\lambda)\right\|(2|\lambda|\|A\|+\|B\|)\right)^{2}+4\left\|\mathrm{R}_{\mathrm{Q}}(\lambda)\right\|\|A\|}}{2\left\|\mathrm{R}_{\mathrm{Q}}(\lambda)\right\|\|A\|}- \\
& -\frac{\left\|\mathrm{R}_{\mathrm{Q}}(\lambda)\right\|(2|\lambda|\|A\|+\|B\|)}{2\left\|\mathrm{R}_{\mathrm{Q}}(\lambda)\right\|\|A\|} .
\end{aligned}
$$

Theorem 1. If $\lambda \in \operatorname{re}(A, B, C)$ and $\lambda_{0} \in \mathbb{C}$, such that $\left|\lambda-\lambda_{0}\right|<\kappa(\lambda)$, then $\lambda_{0} \in \operatorname{re}(A, B, C)$.

Proof. We show that $Q^{-1}\left(\lambda_{0}\right)$ exists and is bounded; we have

$$
\begin{aligned}
\lambda_{0}^{2} A+\lambda_{0} B & +C=\left(\lambda^{2} A+\lambda B+C\right)-\left[\left(\lambda^{2}-\lambda_{0}^{2}\right) A+\left(\lambda-\lambda_{0}\right) B\right]= \\
& \left.=\left(\lambda^{2} A+\lambda B+C\right)\left[I-\mathrm{R}_{\mathrm{Q}}(\lambda)\left(\lambda^{2}-\lambda_{0}^{2}\right) A+\left(\lambda-\lambda_{0}\right) B\right)\right] .
\end{aligned}
$$

Using the condition $\left|\lambda-\lambda_{0}\right|<\kappa(\lambda)$, we obtain

$$
\left\|\mathrm{R}_{\mathrm{Q}}(\lambda)\left(\left(\lambda^{2}-\lambda_{0}^{2}\right) A+\left(\lambda-\lambda_{0}\right) B\right)\right\|<1
$$


By Neumann's theorem (see [1] $\left[I-\mathrm{R}_{\mathrm{Q}}(\lambda)\left(\left(\lambda^{2}-\lambda_{0}^{2}\right) A+\left(\lambda-\lambda_{0}\right) B\right)\right]^{-1}$ exists and it is bounded, i.e.,

$$
\begin{aligned}
& \left\|\left[I-\mathrm{R}_{\mathrm{Q}}(\lambda)\left(\left(\lambda^{2}-\lambda_{0}^{2}\right) A+\left(\lambda-\lambda_{0}\right) B\right)\right]^{-1}\right\| \leqslant \\
& \quad \leqslant\left(1-\left\|\mathrm{R}_{\mathrm{Q}}(\lambda)\left(\left(\lambda^{2}-\lambda_{0}^{2}\right) A+\left(\lambda-\lambda_{0}\right) B\right)\right\|\right)^{-1} .
\end{aligned}
$$

This gives

$$
\mathrm{R}_{\mathrm{Q}}\left(\lambda_{0}\right)=\left[I-\mathrm{R}_{\mathrm{Q}}(\lambda)\left(\left(\lambda^{2}-\lambda_{0}^{2}\right) A+\left(\lambda-\lambda_{0}\right) B\right)\right]^{-1} \mathrm{R}_{\mathrm{Q}}(\lambda),
$$

and

$$
\left\|\mathrm{R}_{\mathrm{Q}}\left(\lambda_{0}\right)\right\| \leqslant\left(1-\left\|\mathrm{R}_{\mathrm{Q}}(\lambda)\left(\left(\lambda^{2}-\lambda_{0}^{2}\right) A+\left(\lambda-\lambda_{0}\right) B\right)\right\|\right)^{-1}\left\|\mathrm{R}_{\mathrm{Q}}(\lambda)\right\| .
$$

Corollary. $\operatorname{sp}(A, B, C)$ is closed in $\mathbb{C}$.

Proof. Using the fact that $\operatorname{re}(A, B, C)$ is the complementary of $\operatorname{sp}(A, B, C)$ in $\mathbb{C}$, and according to the previous theorem, we see that $\operatorname{re}(A, B, C)$ is open in $\mathbb{C}$. $\square$

Theorem 2. $\mathrm{R}_{\mathrm{Q}}(\cdot)$ is analytic in $\operatorname{re}(A, B, C)$ and for all $\lambda \in \operatorname{re}(A, B, C)$ we have

$$
\frac{d \mathrm{R}_{\mathrm{Q}}}{d \lambda}(\lambda)=-\mathrm{R}_{\mathrm{Q}}(\lambda)(2 \lambda A+B) \mathrm{R}_{\mathrm{Q}}(\lambda) .
$$

Proof. For all $\lambda, \lambda_{0} \in \operatorname{re}(A, B, C)$ such that $\left|\lambda-\lambda_{0}\right|<\kappa\left(\lambda_{0}\right)$, we have

$$
\begin{aligned}
\mathrm{R}_{\mathrm{Q}}(\lambda)-\mathrm{R}_{\mathrm{Q}}\left(\lambda_{0}\right) & =\left(\lambda^{2} A+\lambda B+C\right)^{-1}-\left(\lambda_{0}^{2} A+\lambda_{0} B+C\right)^{-1}= \\
& =\mathrm{R}_{\mathrm{Q}}(\lambda)\left(\left(\lambda_{0}^{2}-\lambda^{2}\right) A+\left(\lambda_{0}-\lambda\right) B\right) \mathrm{R}_{\mathrm{Q}}\left(\lambda_{0}\right) .
\end{aligned}
$$

Our goal is to show that

$$
\lim _{\lambda \rightarrow \lambda_{0}}\left\|\frac{\mathrm{R}_{\mathrm{Q}}(\lambda)-\mathrm{R}_{\mathrm{Q}}\left(\lambda_{0}\right)}{\lambda-\lambda_{0}}+\mathrm{R}_{\mathrm{Q}}(\lambda)(2 \lambda A+B) \mathrm{R}_{\mathrm{Q}}(\lambda)\right\|=0 .
$$

We have

$$
\begin{aligned}
& \left\|\frac{\mathrm{R}_{\mathrm{Q}}(\lambda)-\mathrm{R}_{\mathrm{Q}}\left(\lambda_{0}\right)}{\lambda-\lambda_{0}}+\mathrm{R}_{\mathrm{Q}}(\lambda)(2 \lambda A+B) \mathrm{R}_{\mathrm{Q}}(\lambda)\right\|= \\
& =\left\|\frac{\left.\mathrm{R}_{\mathrm{Q}}(\lambda)\left(\lambda_{0}^{2}-\lambda^{2}\right) A+\left(\lambda_{0}-\lambda\right) B\right) \mathrm{R}_{\mathrm{Q}}\left(\lambda_{0}\right)}{\lambda-\lambda_{0}}+\mathrm{R}_{\mathrm{Q}}(\lambda)(2 \lambda A+B) \mathrm{R}_{\mathrm{Q}}(\lambda)\right\|=
\end{aligned}
$$




$$
\begin{aligned}
=\|-\mathrm{R}_{\mathrm{Q}}(\lambda)\left(\left(\lambda_{0}+\lambda\right) A+B\right) \mathrm{R}_{\mathrm{Q}}\left(\lambda_{0}\right)+ & \mathrm{R}_{\mathrm{Q}}(\lambda)(2 \lambda A+B) \mathrm{R}_{\mathrm{Q}}(\lambda) \| \leqslant \\
\leqslant\left|\lambda_{0}+\lambda\right|\left\|\mathrm{R}_{\mathrm{Q}}(\lambda) A\right\|\left\|\mathrm{R}_{\mathrm{Q}}(\lambda)-\mathrm{R}_{\mathrm{Q}}\left(\lambda_{0}\right)\right\|+ & \left\|\mathrm{R}_{\mathrm{Q}}(\lambda) B\right\|\left\|\mathrm{R}_{\mathrm{Q}}(\lambda) ! \mathrm{R}_{\mathrm{Q}}\left(\lambda_{0}\right)\right\|+ \\
& +\left|\lambda-\lambda_{0}\right|\left\|\mathrm{R}_{\mathrm{Q}}(\lambda) A \mathrm{R}_{\mathrm{Q}}(\lambda)\right\| .
\end{aligned}
$$

Using Neumann's theorem, we obtain

$$
\begin{aligned}
& \left\|\mathrm{R}_{\mathrm{Q}}(\lambda)-\mathrm{R}_{\mathrm{Q}}\left(\lambda_{0}\right)\right\|= \\
& =\left\|\mathrm{R}_{\mathrm{Q}}(\lambda)-\sum_{n \geqslant 0} \mathrm{R}_{\mathrm{Q}}(\lambda)^{n}\left(\left(\lambda^{2}-\lambda_{0}^{2}\right) A+\left(\lambda-\lambda_{0}\right) B\right)^{n} \mathrm{R}_{\mathrm{Q}}(\lambda)\right\|= \\
& =\left\|-\sum_{n \geqslant 1} \mathrm{R}_{\mathrm{Q}}(\lambda)^{n}\left(\left(\lambda^{2}-\lambda_{0}^{2}\right) A+\left(\lambda-\lambda_{0}\right) B\right)^{n} \mathrm{R}_{\mathrm{Q}}(\lambda)\right\|= \\
& =\left\|-\sum_{n \geqslant 1} \mathrm{R}_{\mathrm{Q}}(\lambda)^{n}\left(\lambda-\lambda_{0}\right)^{n}\left(\left(\lambda+\lambda_{0}\right) A+B\right)^{n} \mathrm{R}_{\mathrm{Q}}(\lambda)\right\| \leqslant \\
& \leqslant \sum_{n \geqslant 1}\left\|\mathrm{R}_{\mathrm{Q}}(\lambda)\left(\lambda-\lambda_{0}\right)\left(\left(\lambda+\lambda_{0}\right) A+B\right)\right\|^{n}\left\|\mathrm{R}_{\mathrm{Q}}(\lambda)\right\| \leqslant \\
& \leqslant \frac{\left\|\mathrm{R}_{\mathrm{Q}}(\lambda)\right\|\left|\left(\lambda-\lambda_{0}\right)\right|\left\|\left(\left(\lambda+\lambda_{0}\right) A+B\right)\right\|\left\|\mathrm{R}_{\mathrm{Q}}(\lambda)\right\|}{1-\left|\left(\lambda-\lambda_{0}\right)\right|\left\|\left(\left(\lambda+\lambda_{0}\right) A+B\right)\right\|\left\|\mathrm{R}_{\mathrm{Q}}(\lambda)\right\|}
\end{aligned}
$$

Then

$$
\lim _{\lambda \rightarrow \lambda_{0}}\left\|\mathrm{R}_{\mathrm{Q}}(\lambda)-\mathrm{R}_{\mathrm{Q}}\left(\lambda_{0}\right)\right\|=0
$$

We conclude that

$$
\lim _{\lambda \rightarrow \lambda_{0}}\left\|\frac{\mathrm{R}_{\mathrm{Q}}(\lambda)-\mathrm{R}_{\mathrm{Q}}\left(\lambda_{0}\right)}{\lambda-\lambda_{0}}+\mathrm{R}_{\mathrm{Q}}(\lambda)(2 \lambda A+B) \mathrm{R}_{\mathrm{Q}}(\lambda)\right\|=0 .
$$

3. Generalized quadratic spectrum approximation. In this section, we show that the spectral pollution does not appear when we use the generalized quadratic spectrum approximation in our problem. In previous works, Ahues et al in [1] used the $\nu$-convergence of $\left\{A_{n}\right\}_{n \in \mathbb{N}}$ to $A$, i. e.,

- $\sup \left\|A_{n}\right\|<+\infty$,

- $\lim _{n \rightarrow \infty}\left\|\left(A-A_{n}\right) A\right\|=\lim _{n \rightarrow \infty}\left\|\left(A-A_{n}\right) A_{n}\right\|=0$.

To get rid of the spectral pollution problem, we prove the property U, which means: 
If $\left\{A_{n}\right\}_{n \in \mathbb{N}}$ converges to $A$ in $\nu$ - convergence sense, $\lambda_{n} \in \operatorname{sp}\left(A_{n}\right)$ and $\lambda_{n} \rightarrow \lambda$, then $\lambda \in \operatorname{sp}(A)$, we use the collectively compact convergence, i. e., for all $x \in \mathcal{X}, \lim _{n \rightarrow \infty}\left\|\left(A-A_{n}\right) x\right\|_{\mathcal{X}}=0$, and for some positive integer $n_{0}$, the set

$$
\bigcup_{n \geqslant n_{0}}\left\{A_{n} x-A x: x \in \mathcal{X},\|x\|_{\mathcal{X}}=1\right\}
$$

is a relatively compact subset of $\mathcal{X}$. Or, the norm convergence, which means $\lim _{n \rightarrow \infty}\left\|A-A_{n}\right\|=0$.

We must take into consideration that the collectively compact convergence and the norm convergence imply the $\nu$ - convergence but not vice versa: this was proved by Ahues et al in [1].

Also, Guebbai in [8] shows the convergence of the generalized spectrum in the property $\mathrm{U}$ sense, when the sequence of operators $T_{n}, S_{n}$ converges in norm to $T, S$, respectively, and if $\lambda_{n} \in \operatorname{sp}\left(T_{n}, S_{n}\right)$ and $\lambda_{n} \rightarrow \lambda$ then $\lambda \in \operatorname{sp}(T, S)$. Therefore, Khellaf et al [11], [13] treated the same problem presented in $[8]$ in the sense of property U using the collectively compact convergence of $T_{n}$ and $S_{n}$.

Our interest to those two convergence forms results from numerical methods developed to approximate integral operators, such as the projection method, which generally converges in the norm sense for compact operators, and the collectively compact convergence is obtained for Nystöm (see Atkinson et al [3], Ahues et al [1]).

We rewrite the property $\mathrm{U}$ for our problem as follows:

If $A_{n}, B_{n}$ and $C_{n}$ converge to $A, B$, and $C$, respectively, in a given sense, $\lambda_{n} \in \operatorname{sp}\left(A_{n}, B_{n}, C_{n}\right)$, and $\lambda_{n} \rightarrow \lambda$, then $\lambda \in \operatorname{sp}(A, B, C)$.

Let $A, \tilde{A}, B, \tilde{B}, C$ and $\tilde{C}$ be in $\operatorname{BL}(\mathcal{X})$; define $\tilde{Q}(\lambda)$ as follows:

$$
\tilde{Q}(\lambda)=\lambda^{2} \tilde{A}+\lambda \tilde{B}+\tilde{C} .
$$

Theorem 3. Let $\lambda \in \operatorname{re}(A, B, C)$ be such that

$$
\left\|[Q(\lambda)-\tilde{Q}(\lambda)] \mathrm{R}_{Q}(\lambda)\right\|<1 \text { or }\left\|\left[(Q(\lambda)-\tilde{Q}(\lambda)) \mathrm{R}_{Q}(\lambda)\right]^{2}\right\|<1 .
$$

Then $\lambda \in \operatorname{re}(\tilde{A}, \tilde{B}, \tilde{C})$, and

$$
\left\|\mathrm{R}_{\tilde{\mathrm{Q}}}(\lambda)\right\| \leqslant \frac{\left\|\mathrm{R}_{\mathrm{Q}}(\lambda)\right\|}{1-\left\|(Q(\lambda)-\tilde{Q}(\lambda)) \mathrm{R}_{\mathrm{Q}}(\lambda)\right\|},
$$




$$
\left\|\mathrm{R}_{\tilde{Q}}(\lambda)\right\| \leqslant \frac{\left\|\mathrm{R}_{\mathrm{Q}}(\lambda)\right\|\left[1+\left\|\left[(Q(\lambda)-\tilde{Q}(\lambda)) \mathrm{R}_{\mathrm{Q}}(\lambda)\right]^{2}\right\|\right]}{1-\left\|\left[(Q(\lambda)-\tilde{Q}(\lambda)) \mathrm{R}_{\mathrm{Q}}(\lambda)\right]^{2}\right\|},
$$

respectively.

Proof. We can write

$$
\begin{aligned}
\tilde{Q}(\lambda) & =\tilde{Q}(\lambda)-Q(\lambda)+Q(\lambda)= \\
& =Q(\lambda)\left[I-(Q(\lambda)-\tilde{Q}(\lambda)) \mathrm{R}_{\mathrm{Q}}(\lambda)\right] .
\end{aligned}
$$

Using Neumann's theorem under the first condition in Theorem 3, we see that $\left[I-(Q(\lambda)-\tilde{Q}(\lambda)) \mathrm{R}_{Q}(\lambda)\right]^{-1}$ exists and is bounded, i. e.,

$$
\left\|\left[I-(Q(\lambda)-\tilde{Q}(\lambda)) \mathrm{R}_{Q}(\lambda)\right]^{-1}\right\| \leqslant\left(1-\left\|(Q(\lambda)-\tilde{Q}(\lambda)) \mathrm{R}_{Q}(\lambda)\right\|\right)^{-1} .
$$

It follows that

$$
\begin{gathered}
\mathrm{R}_{\tilde{\mathrm{Q}}}(\lambda)=\left[I-(Q(\lambda)-\tilde{Q}(\lambda)) \mathrm{R}_{\mathrm{Q}}(\lambda)\right]^{-1} \mathrm{R}_{\mathrm{Q}}(\lambda), \\
\left\|\mathrm{R}_{\tilde{Q}}(\lambda)\right\| \leqslant \frac{\left\|\mathrm{R}_{\mathrm{Q}}(\lambda)\right\|}{1-\left\|(Q(\lambda)-\tilde{Q}(\lambda)) \mathrm{R}_{\mathrm{Q}}(\lambda)\right\|} .
\end{gathered}
$$

On the other hand, we have

$$
\begin{gathered}
\mathrm{R}_{\tilde{Q}}(\lambda)=\mathrm{R}_{\mathrm{Q}}(\lambda) \sum_{k=0}^{\infty}\left[(Q(\lambda)-\tilde{Q}(\lambda)) \mathrm{R}_{\mathrm{Q}}(\lambda)\right]^{2 k}+ \\
+\mathrm{R}_{\mathrm{Q}}(\lambda) \sum_{k=0}^{\infty}\left[(Q(\lambda)-\tilde{Q}(\lambda)) \mathrm{R}_{\mathrm{Q}}(\lambda)\right]^{2 k+1}= \\
=\mathrm{R}_{\mathrm{Q}}(\lambda)\left[I+(Q(\lambda)-\tilde{Q}(\lambda)) \mathrm{R}_{\mathrm{Q}}(\lambda)\right] \sum_{k=0}^{\infty}\left[\left((Q(\lambda)-\tilde{Q}(\lambda)) \mathrm{R}_{\mathrm{Q}}(\lambda)\right)^{2}\right]^{k} .
\end{gathered}
$$

This gives

$$
\left\|\mathrm{R}_{\tilde{Q}}(\lambda)\right\| \leqslant \frac{\left\|\mathrm{R}_{Q}(\lambda)\right\|\left[1+\left\|\left[(Q(\lambda)-\tilde{Q}(\lambda)) \mathrm{R}_{Q}(\lambda)\right]^{2}\right\|\right]}{1-\left\|\left[(Q(\lambda)-\tilde{Q}(\lambda)) \mathrm{R}_{Q}(\lambda)\right]^{2}\right\|} .
$$


The proof of Theorem 3 is complete.

Lemma 1. Let $\left\{N_{n}\right\}_{n \in \mathbb{N}},\left\{M_{n}\right\}_{n \in \mathbb{N}} \subset \mathrm{BL}(\mathcal{X})$, and $N, M, K \in \mathrm{BL}(\mathcal{X})$. If $N_{n}$ and $M_{n}$ converge to $N$ and $M$, respectively, in the collectively compact sense; then

$$
\lim _{n \rightarrow \infty}\left\|\left(N-N_{n}\right) K\left(M-M_{n}\right)\right\|=\lim _{n \rightarrow \infty}\left\|\left(M-M_{n}\right) K\left(N-N_{n}\right)\right\|=0 .
$$

Proof. Using the collectively compact convergence of $\left\{N_{n}\right\}_{n \in \mathbb{N}},\left\{M_{n}\right\}_{n \in \mathbb{N}}$, we have

For all $x \in \mathcal{X}, \lim _{n \rightarrow \infty}\left\|\left(N-N_{n}\right) x\right\|_{\mathcal{X}}=0$ and there is an integer $n_{1} \in \mathbb{N}$ such that

$$
S_{1}=\bigcup_{n \geqslant n_{1}}\left\{N_{n} x-N x: x \in \mathcal{X},\|x\|_{\mathcal{X}}=1\right\}
$$

is relatively compact.

For all $x \in \mathcal{X}, \lim _{n \rightarrow \infty}\left\|\left(M-M_{n}\right) x\right\|_{\mathcal{X}}=0$ and there is an integer $n_{2} \in \mathbb{N}$ such that

$$
S_{2}=\bigcup_{n \geqslant n_{2}}\left\{M_{n} x-M x: x \in X,\|x\|_{\mathcal{X}}=1\right\}
$$

has a compact closure in $X$.

Also, $K\left(S_{1}\right)$ and $K\left(S_{2}\right)$ are relatively compact; therefore, we obtain

$$
\begin{aligned}
\left\|\left(N-N_{n}\right) K\left(M-M_{n}\right)\right\| & =\sup _{\|x\|_{\mathcal{X}}=1}\left\|\left(N-N_{n}\right) K\left(M-M_{n}\right) x\right\|_{\mathcal{X}}= \\
& =\sup _{\|x\|_{\mathcal{X}}=1}\left\|\left(N-N_{n}\right) K\left(M x-M_{n} x\right)\right\|_{\mathcal{X}}= \\
& =\sup _{y \in K\left(S_{2}\right)}\left\|\left(N-N_{n}\right) y\right\|_{\mathcal{X}} \rightarrow 0 .
\end{aligned}
$$

Let $\left\{A_{n}\right\}_{n \in \mathbb{N}},\left\{B_{n}\right\}_{n \in \mathbb{N}}$, and $\left\{C_{n}\right\}_{n \in \mathbb{N}} \subset \mathrm{BL}(\mathcal{X})$. We define the following hypothesis

(H1) $A_{n}, B_{n}$, and $C_{n}$ converge to $A, B$, and $C$, respectively, in the norm sense.

(H2) $A_{n}, B_{n}$, and $C_{n}$ converge in the collectively compact sense to $A, B$, and $C$, respectively. 
Theorem 4. Under the assumptions (H1) or (H2) and for all $n \in \mathbb{N}$, if $\lambda_{n} \in \operatorname{sp}\left(A_{n}, B_{n}, C_{n}\right)$ and $\lambda_{n} \rightarrow \lambda$ then $\lambda \in \operatorname{sp}(A, B, C)$.

Proof. In the first part, we suppose that $\lambda \in \operatorname{re}(A, B, C)$ and $\left\{A_{n}\right\}_{n \in \mathbb{N}}$, $\left\{B_{n}\right\}_{n \in \mathbb{N}}$ and $\left\{C_{n}\right\}_{n \in \mathbb{N}}$ satisfy $(H 1)$. We know that $\operatorname{re}(A, B, C)$ is an open set in $\mathbb{C}$, i. e., there is $\delta>0$ such that

$$
\Omega:=\left\{\lambda_{0} \in \mathbb{C}:\left|\lambda_{0}-\lambda\right| \leqslant \delta\right\} \subset \operatorname{re}(A, B, C) .
$$

But, $\mathrm{R}_{\mathrm{Q}}(\cdot)$ is analytic on $\operatorname{re}(A, B, C)$; so, we can define $\beta$ as follows:

$$
\beta:=\sup \left\{\left\|R_{Q}\left(\lambda_{0}\right)\right\|: \lambda_{0} \in \Omega\right\}<\infty .
$$

For all $\lambda_{0} \in \Omega$, there are $n_{1}, n_{2}$ and $n_{3}$, such that

$$
\begin{gathered}
\left\|A-A_{n}\right\| \leqslant \frac{1}{6 \beta(\delta+|\lambda|)^{2}}, \quad n \geqslant n_{1}, \\
\left\|B-B_{n}\right\| \leqslant \frac{1}{6 \beta(\delta+|\lambda|)}, \quad n \geqslant n_{2}, \\
\left\|C-C_{n}\right\| \leqslant \frac{1}{6 \beta}, \quad n \geqslant n_{3} ;
\end{gathered}
$$

then, for all $n \geqslant \max \left(n_{1}, n_{2}, n_{3}\right)$, we obtain

$$
\begin{aligned}
& \left\|\left[Q\left(\lambda_{0}\right)-Q_{n}\left(\lambda_{0}\right)\right] \mathrm{R}_{\mathrm{Q}}\left(\lambda_{0}\right)\right\|= \\
= & \left\|\left[\lambda_{0}^{2}\left(A-A_{n}\right)+\lambda_{0}\left(B-B_{n}\right)+\left(C-C_{n}\right)\right] \mathrm{R}_{\mathrm{Q}}\left(\lambda_{0}\right)\right\| \leqslant \\
\leqslant & \left(\left|\lambda_{0}\right|^{2}\left\|A-A_{n}\right\|+\left|\lambda_{0}\right|\left\|B-B_{n}\right\|+\left\|C-C_{n}\right\|\right) \beta \leqslant \\
\leqslant & \frac{1}{6}+\frac{1}{6}+\frac{1}{6}=\frac{1}{2},
\end{aligned}
$$

using Theorem 3 with the first condition. So, $R_{Q_{n}}\left(\lambda_{0}\right)$ exists and it is bounded; therefore, $\Omega \subset \operatorname{re}\left(A_{n}, B_{n}, C_{n}\right)$ for all $n$ large enough. As though $\lambda_{n} \rightarrow \lambda$, we have $\lambda_{n} \in \Omega \subset \operatorname{re}\left(A_{n}, B_{n}, C_{n}\right)$ for all $n$; this contradicts to the assumption that $\lambda_{n} \in \operatorname{sp}\left(A_{n}, B_{n}, C_{n}\right)$ for each $n$. Therefore, $\lambda$ must be in $\operatorname{sp}(A, B, C)$.

In the second part, we suppose that $\left\{A_{n}\right\}_{n \in \mathbb{N}},\left\{B_{n}\right\}_{n \in \mathbb{N}}$ and $\left\{C_{n}\right\}_{n \in \mathbb{N}}$ satisfy ( $\mathrm{H} 2)$. We follow the same reasoning as in the previous part. For all $\lambda_{0} \in \Omega$, there are $n_{1}, n_{2}, n_{3}, n_{4}, n_{5}, n_{6}, n_{7}, n_{8}$, and $n_{9}$, such that

$$
\left\|\left(A-A_{n}\right) \mathrm{R}_{\mathrm{Q}}\left(\lambda_{0}\right)\left(A-A_{n}\right)\right\| \leqslant \frac{1}{18 \beta\left(\delta+|\lambda|^{4}\right)}, \quad n \geqslant n_{1},
$$




$$
\begin{aligned}
& \left\|\left(B-B_{n}\right) \mathrm{R}_{\mathrm{Q}}\left(\lambda_{0}\right)\left(A-A_{n}\right)\right\| \leqslant \frac{1}{18 \beta\left(\delta+|\lambda|^{3}\right)}, \quad n \geqslant n_{2}, \\
& \left\|\left(A-A_{n}\right) \mathrm{R}_{\mathrm{Q}}\left(\lambda_{0}\right)\left(B-B_{n}\right)\right\| \leqslant \frac{1}{18 \beta\left(\delta+|\lambda|^{3}\right)}, \quad n \geqslant n_{3}, \\
& \left\|\left(B-B_{n}\right) \mathrm{R}_{\mathrm{Q}}\left(\lambda_{0}\right)\left(B-B_{n}\right)\right\| \leqslant \frac{1}{18 \beta\left(\delta+|\lambda|^{2}\right)}, \quad n \geqslant n_{4}, \\
& \left\|\left(A-A_{n}\right) \mathrm{R}_{\mathrm{Q}}\left(\lambda_{0}\right)\left(C-C_{n}\right)\right\| \leqslant \frac{1}{18 \beta\left(\delta+|\lambda|^{2}\right)}, \quad n \geqslant n_{5}, \\
& \left\|\left(C-C_{n}\right) \mathrm{R}_{\mathrm{Q}}\left(\lambda_{0}\right)\left(A-A_{n}\right)\right\| \leqslant \frac{1}{18 \beta\left(\delta+|\lambda|^{2}\right)}, \quad n \geqslant n_{6}, \\
& \left\|\left(B-B_{n}\right) \mathrm{R}_{\mathrm{Q}}\left(\lambda_{0}\right)\left(C-C_{n}\right)\right\| \leqslant \frac{1}{18 \beta(\delta+|\lambda|)}, \quad n \geqslant n_{7}, \\
& \left\|\left(C-C_{n}\right) \mathrm{R}_{\mathrm{Q}}\left(\lambda_{0}\right)\left(B-B_{n}\right)\right\| \leqslant \frac{1}{18 \beta(\delta+|\lambda|)}, \quad n \geqslant n_{8}, \\
& \left\|\left(C-C_{n}\right) \mathrm{R}_{\mathrm{Q}}\left(\lambda_{0}\right)\left(C-C_{n}\right)\right\| \leqslant \frac{1}{18 \beta}, \quad n \geqslant n_{9} .
\end{aligned}
$$

Then, for all $n \geqslant \max \left(n_{1}, n_{2}, n_{3}, n_{4}, n_{5}, n_{6}, n_{7}, n_{8}, n_{9}\right)$, we obtain

$$
\begin{aligned}
& \left\|\left[\left(Q\left(\lambda_{0}\right)-Q_{n}\left(\lambda_{0}\right)\right) R_{Q}\left(\lambda_{0}\right)\right]^{2}\right\| \leqslant \beta\left(\left\|\left(C-C_{n}\right) \mathrm{R}_{\mathrm{Q}}\left(\lambda_{0}\right)\left(C-C_{n}\right)\right\|+\right. \\
+ & \left|\lambda_{0}\right|^{4}\left\|\left(A-A_{n}\right) \mathrm{R}_{\mathrm{Q}}\left(\lambda_{0}\right)\left(A-A_{n}\right)\right\|+\left|\lambda_{0}\right|^{3}\left\|\left(B-B_{n}\right) \mathrm{R}_{\mathrm{Q}}\left(\lambda_{0}\right)\left(A-A_{n}\right)\right\|+ \\
+ & \left|\lambda_{0}\right|^{3}\left\|\left(A-A_{n}\right) \mathrm{R}_{\mathrm{Q}}\left(\lambda_{0}\right)\left(B-B_{n}\right)\right\|+\left|\lambda_{0}\right|^{2}\left\|\left(B-B_{n}\right) \mathrm{R}_{\mathrm{Q}}\left(\lambda_{0}\right)\left(B-B_{n}\right)\right\|+ \\
+ & \left|\lambda_{0}\right|^{2}\left\|\left(A-A_{n}\right) \mathrm{R}_{\mathrm{Q}}\left(\lambda_{0}\right)\left(C-C_{n}\right)\right\|+\left|\lambda_{0}\right|^{2}\left\|\left(C-C_{n}\right) \mathrm{R}_{\mathrm{Q}}\left(\lambda_{0}\right)\left(A-A_{n}\right)\right\|+ \\
+ & \left.\left|\lambda_{0}\right|\left\|\left(B-B_{n}\right) \mathrm{R}_{\mathrm{Q}}\left(\lambda_{0}\right)\left(C-C_{n}\right)\right\|+\left|\lambda_{0}\right|\left\|\left(C-C_{n}\right) \mathrm{R}_{\mathrm{Q}}\left(\lambda_{0}\right)\left(B-B_{n}\right)\right\|\right) \leqslant \\
\leqslant & \frac{1}{18}+\frac{1}{18}+\frac{1}{18}+\frac{1}{18}+\frac{1}{18}+\frac{1}{18}+\frac{1}{18}+\frac{1}{18}+\frac{1}{18}=\frac{1}{2} .
\end{aligned}
$$

Using the second condition in Theorem 3, we have $\lambda_{n} \in \operatorname{re}\left(A_{n}, B_{n}, C_{n}\right)$ for all $n$ : this contradicts to the assumption that $\lambda_{n} \in \operatorname{sp}\left(A_{n}, B_{n}, C_{n}\right)$ for each $n$. Therefore, $\lambda$ must be in $\operatorname{sp}(A, B, C)$.

4. Numerical tests. In this section, we introduce a numerical example to validate our theoretical results.

Example construction. In order to construct numerical tests, we choose to study the quadratic pencil of Schrödinger's operator presented by Bairamov et al [4], Krall et al [14]. This problem is basically defined 
in $L^{2}([0,+\infty[)$ as follows:

$$
(P)\left\{\begin{array}{l}
-y^{\prime \prime}+\left(V(x)+2 \lambda U(x)-\lambda^{2}\right) y=0, x \in[0,+\infty[ \\
y(0)=0
\end{array}\right.
$$

where, $V, U$ are complex functions. The authors in [4], [14] used analytical technics to obtain a localization of the spectrum.

In our example, we choose $V(x)=x^{2}$ and $U(x)=-\frac{1}{2}$, to obtain the similar problem to $(P)$, as follows:

$$
\left(P^{\prime}\right)\left\{\begin{array}{l}
-y^{\prime \prime}+\left(x^{2}-\lambda+\lambda^{2}\right) y=0 \\
y(0)=0
\end{array}\right.
$$

To study the generalized quadratic spectrum better, we introduce the unbounded operator $S$ of the form:

$$
S y=-y^{\prime \prime}+x^{2} y, x \in[0,+\infty[,
$$

where $S$ is the harmonic oscillator, which is defined as

$$
\mathcal{D}(S)=H^{2}\left(\left[0,+\infty[) \cap\left\{\varphi \in L ^ { 2 } \left(\left[0,+\infty[): \int_{0}^{+\infty} x^{2}|\varphi(x)|^{2} d x<+\infty\right\}\right.\right.\right.\right.
$$

into $L^{2}\left(\left[0,+\infty[)\right.\right.$. We define the Laplacian operator denoted by $L_{p}$ as follows:

$$
\begin{aligned}
L_{p}: \mathcal{D}\left(L_{p}\right) & \rightarrow L^{2}(0, a), \\
y & \mapsto L_{p} y=-y^{\prime \prime} .
\end{aligned}
$$

Here $\mathcal{D}\left(L_{p}\right)=\left\{y \in H^{2}(0, a), y(0)=y(a)=0\right\}$. In addition, we define its inverse denoted $A_{a}$ as:

$$
A_{a} y(x)=\int_{0}^{a} K_{a}(x, t) y(t) d t
$$

which is bounded and linear in $L^{2}(0, a)$, and for all $y \in L^{2}(0, a)$

$$
B_{a} y(x)=-\int_{0}^{a} K_{a}(x, t) y(t) d t
$$




$$
C_{a} y(x)=y(x)+\int_{0}^{a} K_{a}(x, t) t^{2} y(t) d t,
$$

where $K_{a}$ is the Green kernel (see Roach [18]), by

$$
K_{a}(x, t)= \begin{cases}\frac{x(a-t)}{a} & \text { if } 0 \leqslant x \leqslant t \leqslant a, \\ \frac{t(a-x)}{a} & \text { if } 0 \leqslant t \leqslant x \leqslant a .\end{cases}
$$

For all $\mu \in \mathbb{C}$ and all $y \in \mathcal{D}(S)-\{0\}$, we present the eigenvalue problem corresponding to $S$ by:

$$
S y=\mu y,
$$

which can be presented as follows:

$$
\left(P^{\prime \prime}\right)\left\{\begin{array}{l}
-y^{\prime \prime}+x^{2} y=\mu y \\
y(0)=0
\end{array}\right.
$$

We see that $\left(P^{\prime \prime}\right)$ is equivalent to $\left(P^{\prime}\right)$ with $\mu=\left(\lambda-\lambda^{2}\right)$; the eigenvalues of the operator $S$ are $\left\{4 n-1, n \in \mathbb{N}^{*}\right\}$. As a result, we find that $\lambda=\frac{1}{2} \pm i \frac{\sqrt{16 n-5}}{2}, n \in \mathbb{N}^{*}$ in $\left(P^{\prime}\right)$.

We define the operator $S_{a}$ for all $a>0$ by

$$
S_{a} y=-y^{\prime \prime}+x^{2} y, y \in \mathcal{D}\left(S_{a}\right)=H^{2}(0, a) .
$$

The eigenvalue problem $S_{a} y=\mu y$ is equivalent to: Find $\mu \in \mathbb{C}$ and $y \in \mathcal{D}\left(S_{a}\right)-\{0\}$ satisfying

$$
\left(P_{a}^{\prime}\right)\left\{\begin{array}{l}
-y^{\prime \prime}+x^{2} y=\mu y \\
y(0)=y(a)=0
\end{array}\right.
$$

The authors in [9], [11] proved that for all $a>0, \operatorname{sp}\left(S_{a}\right) \subset \operatorname{sp}(S)$ and $\operatorname{sp}(S)=\bigcup_{a>0} \operatorname{sp}\left(S_{a}\right)$.

Theorem 5. The eigenvalue problem $\left(P_{a}^{\prime}\right)$ is equivalent to the following associated quadratic eigenvalue problem $\left(Q_{a}\right)$, i. e., $\lambda$ is a quadratic eigenvalue of $S_{a}$ if and only if $\lambda$ is a generalized quadratic eigenvalue of three bounded operators $\left(A_{a}, B_{a}, C_{a}\right)$. Here

$$
\left(Q_{a}\right): Q_{a}(\lambda) y:=\lambda^{2} A_{a} y+\lambda B_{a} y+C_{a} y=0 .
$$


Proof. Let $\lambda$ be a quadratic eigenvalue of $S_{a}$, and $y \in \mathcal{D}\left(S_{a}\right)$ be the associated eigenvector, i. e.,

$$
S_{a} y=\left(\lambda-\lambda^{2}\right) y \text {. }
$$

We have

$$
L_{p} y+x^{2} y-\lambda y+\lambda^{2} y=0 .
$$

Applying $A_{a}$, we obtain

$$
\lambda^{2} A_{a} y+\lambda B_{a} y+C_{a} y=0 .
$$

As a result, $\lambda$ is a generalized quadratic eigenvalue of $\left(A_{a}, B_{a}, C_{a}\right)$. Conversely, let $\lambda$ be a generalized quadratic eigenvalue of $\left(A_{a}, B_{a}, C_{a}\right)$, and $y \in L^{2}(0, a)-\{0\}$ be the eigenvector associate to $\lambda$, i. e.,

$$
\lambda^{2} A_{a} y+\lambda B_{a} y+C_{a} y=0 .
$$

Then we have

$$
y=A_{a}\left(\lambda y-\lambda^{2} y-x^{2} y\right) .
$$

It is clear that $\left(\lambda y-\lambda^{2} y-x^{2} y\right) \in L^{2}(0, a)$; then $y \in \mathcal{D}\left(L_{p}\right)$. Applying $L_{p}$, we have

$$
\begin{gathered}
L_{p} y=\lambda y-\lambda^{2} y-x^{2} y \\
\int_{0}^{a} x^{2}|y(x)| d x \leqslant|\lambda|^{2}\|y\|_{L^{2}(0, a)}+|\lambda|\|y\|_{L^{2}(0, a)}+\left\|L_{p} y\right\|_{L^{2}(0, a)}<+\infty,
\end{gathered}
$$

we conclude that $y \in \mathcal{D}\left(S_{a}\right)-\{0\}$, and

$$
S_{a} y=\left(\lambda-\lambda^{2}\right) y,
$$

finally, $\lambda$ is a quadratic eigenvalue of $S_{a}$.

Numerical processing. In this section, we build a Nyström method to approximate the operators $A_{a}, B_{a}$, and $C_{a}$. Atkinson in [3] showed that this method converges in collectively compact sense for our case.

We define a subdivision of $[0, a]$ as follows: for all $n \geqslant 1$,

$$
h=\frac{a}{n}, \quad t_{i}=(i-1) h, 1 \leqslant i \leqslant n+1 .
$$


We define the sequences of operators $\left\{A_{n}\right\}_{n \in \mathbb{N}^{*}},\left\{B_{n}\right\}_{n \in \mathbb{N}^{*}}$ and $\left\{C_{n}\right\}_{n \in \mathbb{N}^{*}}$ by: $\forall y \in \mathcal{D}\left(S_{a}\right)$

$$
\begin{aligned}
& A_{n} y(x)=\sum_{i=1}^{n+1} \omega_{i} K_{a}\left(x, t_{i}\right) y\left(t_{i}\right), \\
& B_{n} y(x)=-\sum_{i=1}^{n+1} \omega_{i} K_{a}\left(x, t_{i}\right) y\left(t_{i}\right), \\
& C_{n} y(x)=y(x)+\sum_{i=1}^{n+1} \omega_{i} K_{a}\left(x, t_{i}\right) t_{i}^{2} y\left(t_{i}\right) .
\end{aligned}
$$

Here $\omega_{1}=\omega_{n+1}=\frac{h}{2}$ and $\omega_{i}=h$ for $2 \leqslant i \leqslant n$; this is known as the trapezoidal rule. It is one of the quadrature approximation methods presented by Argyros, Regmi in [2] and [17].

Therefore, when we replace $x$ by $t_{j}$ for $1 \leqslant j \leqslant n+1$, we get the following quadratic eigenvalue matrix problem:

$$
\lambda_{n}^{2} M_{A_{a}} v+\lambda_{n} M_{B_{a}} v+M_{C_{a}} v=0,
$$

where

$$
\begin{aligned}
& M_{A_{a}}(i, j)=\omega_{i} K_{a}\left(t_{j}, t_{i}\right) \\
& M_{B_{a}}(i, j)=-\omega_{i} K_{a}\left(t_{j}, t_{i}\right) \\
& M_{C_{a}}(i, j)=I_{n+1}(i, j)+\omega_{i} K_{a}\left(t_{j}, t_{i}\right) t_{i}^{2}
\end{aligned}
$$

$I_{n+1}$ is the identity matrix of order $n+1$ and $v$ is the quadratic eigenvector.

To present our numerical results, we introduce this definition:

$\lambda_{n}$ is said to be $\varepsilon$-acceptable eigenvalue if $\operatorname{dist}\left(\lambda_{n}, \operatorname{sp}\left(S_{a}\right)\right)<\varepsilon$.

Also, we set:

- $V P_{a p p}(n)$ is the number of approximate eigenvalues obtained for $n \geqslant 1$

- $V P_{a c c}(n)$ is the number of $\varepsilon$ - acceptable eigenvalues obtained for $n \geqslant 1$

- $P \%=V P_{a c c}(n) / V P_{a p p}(n) \%$.

Numerical tests are made for different values of $n$ with fixed $\varepsilon$ and $a$.

In the first case, we fixe $\varepsilon=10^{-2}$ and $a=1$. The numerical results are presented in Table 1. 
5. Conclusion. The main aim of our studies in this paper has been to build an approximate method for solving the spectral pollution problem and to show the convergence of the generalized quadratic spectrum in the property U-sense under the norm convergence and the collectively compact convergence. We proved the equivalence between the quadratic pencil of the Schrödinger's operator (unbounded operator) and the quadratic spectral problem in the case of three bounded operators.

Our numerical tests showed the effectiveness of the generalized quadratic spectrum approximation method basing in the Nyström method. Percentage increase of the $\varepsilon$-acceptable eigenvalues showed that the spectral pollution is avoided by our method.

In the second case, we choose $\varepsilon=10^{-3}$ and $a=1$. The numerical results are presented in Table 2.

\begin{tabular}{cccc}
\hline$n$ & $V P_{a p p}$ & $V P_{a c c}$ & $P \%$ \\
\hline 100 & 198 & 166 & $83 \%$ \\
200 & 398 & 374 & $93 \%$ \\
300 & 598 & 570 & $95 \%$ \\
400 & 798 & 764 & $95 \%$ \\
500 & 998 & 974 & $97 \%$ \\
\hline
\end{tabular}

Table 1: Percentage of the $10^{-2}-$ acceptable eigenvalues.

\begin{tabular}{cccc}
\hline$n$ & $V P_{a p p}$ & $V P_{a c c}$ & $P \%$ \\
\hline 100 & 198 & 20 & $10 \%$ \\
200 & 398 & 106 & $26 \%$ \\
300 & 598 & 232 & $38 \%$ \\
400 & 798 & 400 & $50 \%$ \\
500 & 998 & 632 & $63 \%$ \\
600 & 1198 & 860 & $71 \%$ \\
700 & 1398 & 1074 & $76 \%$ \\
800 & 1598 & 1276 & $79 \%$ \\
900 & 1798 & 1466 & $81 \%$ \\
1000 & 1998 & 1682 & $84 \%$ \\
1200 & 2398 & 2074 & $86 \%$ \\
1400 & 2798 & 2458 & $87 \%$ \\
2000 & 3998 & 3678 & $92 \%$ \\
2500 & 4998 & 4688 & $93.8 \%$ \\
\hline
\end{tabular}

Table 2: Percentage of the $10^{-3}-$ acceptable eigenvalues. 
Acknowledgements. We appreciate editorial board and the reviewer for your precious time in reviewing our paper and providing valuable comments that have significantly improved our paper.

\section{References}

[1] Ahues M., Largillier A., Limaye B. Computations For Bounded Operators. CRC Press., 2001.

[2] Argyros I. K., Regmi S. Undergraduate Research at Cameron University on Iterative Procedures in Banach and Other Spaces. Nova Science Publisher. New York, USA., 2019.

[3] Atkinson K. E. The Numerical Solution of Integral Equations of The Second Kind. Cambridge University Press., 1996.

[4] Bairamov E., Çakar Ö., Çelebi A. O. Quadratic Pencil of Schrödinger Operators With Spectral Singularities: Discrete Spectrum And Principal Functions. Journal of Mathematical Analysis and Applications., 1997, vol. 216, no. 1 , pp. $303-320$.

DOI: https://doi.org/10.1006/jmaa.1997.5689

[5] Boulton L. Spectral Pollution And Eigenvalue Bounds. Applied Numerical Mathematics., 2016, vol. 99, pp. 1-23.

DOI: https://doi.org/10.1016/j. apnum.2015.08.007

[6] Chen C., Ma C. An Accelerated Cyclic-Reduction-Based Solvent Method For Solving Quadratic Eigenvalue Problem of Gyroscopic Systems. Computers and Mathematics with Applications., 2019, vol. 77, no. 10, pp. 2585-2595. DOI: https://doi.org/10.1016/j . camwa.2018.12.040

[7] Davies, E. B., Plum, M. Spectral Pollution. IMA journal of numerical analysis., 2004, vol. 24 , no. 3, pp. $417-438$.

DOI: https://doi.org/10.1093/imanum/24.3.417

[8] Guebbai H. Generalized Spectrum Approximation And Numerical Computation of Eigenvalues For Schrödinger's Operators. Lobachevskii Journal of Mathematics., 2013, vol. 34, no. 1, pp. 45-60.

DOI: https://doi.org/10.1134/S1995080213010058

[9] Guebbai H., Largillier A. Spectra And Pseudo Spectra of a ConvectionDiffusion Operator. Integral Methods in Science and Engineering., 2011, pp. 173-180. DOI: https://doi.org/10.1007/978-0-8176

[10] Huang T. M., Lin W. W., Tian H., Chen G. H. The Full Spectrum of Large Sparse Palindromic Quadratic Eigenvalue Problems Arising From Surface Green's Function Calculations. Journal of Computational Physics., 2018, vol. 356 , pp. $340-355$.

DOI: https://doi.org/10.1016/j.jcp.2017.12.011 
[11] Khellaf A., Guebbai H., Lemita S., Aissaoui M. Z. Eigenvalues Computation by The Generalized Spectrum Method of Schrödinger's Operator. Computational and Applied Mathematics., 2018, vol. 37, no. 5, pp. 5965-5980. DOI:10.1007/s40314-018-0673-8.

[12] Khellaf A., Merchela W., Guebbai H. New Sufficient Conditions For The Computation of Generalized Eigenvalues. Russian Mathematics., 2021, vol. 65 , no. 2 , pp. $65-68$.

DOI: https://doi.org/10.3103/S1066369X21020067

[13] Khellaf A., Guebbai H. A Note On Generalized Spectrum Approximation. Lobachevskii Journal of Mathematics., 2018, vol. 39, no. 9, pp. 1388-1395. DOI: https://doi.org/0.1134/S1995080218090263

[14] Krall A. M., Bairamov E., Çakar Ö. Spectrum and Spectral Singularities of a Quadratic Pencil of a Schrödinger Operator With a General Boundary Condition. Journal of Differential Equations., 1999, vol. 151, no. 2, pp. 252 -267. DOI: https://doi.org/10.1006/jdeq.1998.3519

[15] Llobet X., Appert K., Bondeson A., Vaclavik J. On Spectral Pollution. Computer physics communications., 1990, vol. 59, no. 2, pp. 199-216. DOI: https://doi.org/10.1016/0010-4655(90)90170-6

[16] Rappaz J., Hubert J. S., Palencia E. S., Vassiliev D. On Spectral Pollution in The Finite Element Approximation of Thin Elastic "membrane" Shells. Numerische Mathematik., 1997, vol. 75, no. 4, pp. 473-500.

DOI: https://doi.org/10.1007/s002110050249

[17] Regmi S. Optimized Iterative Methods with Applications in Diverse Disciplines. Nova Science Publisher. New York, USA., 2021.

[18] Roach G. F. Green's Functions. Cambridge Univ. Press., 1982.

[19] Tisseur F., Meerbergen K. The Quadratic Eigenvalue Problem. SIAM review, 2001, vol. 43, no. 2 , pp. $235-286$.

DOI: https://doi.org/10.1137/S0036144500381988

Received March 30, 2021.

In revised form, June 14, 2021.

Accepted June 16, 2021.

Published online July 1, 2021.

Laboratoire des Mathématiques Appliquées et de Modélisation, Université 8 mai 1945 guelma. B.P.401 Guelma 24000 Algérie.

S. Kamouche: kamouche.somia@univ-guelma.dz; soumia.kamouche@gmail.com

H. Guebbai: guebbai.hamza@univ-guelma.dz; guebaihamza@yahoo.fr

M. Ghiat: mourad.ghi24@gmail.com, ghiat.mourad@univ-guelma.dz

S. Segni: segni.anis@gmail.com, segni.sami@univ-guelma.dz 